\title{
Corpus-based typology analysis of abbreviations in the European Commission's Mobility related documents
}

\author{
Ivo FABIJANIĆ ${ }^{1}$, Frane MALENICA ${ }^{2}$
}

In this paper, we provide a typological overview of abbreviations found in the corpus of Erasmus+ mobility documents, presented as an overview of classification and taxonomic arrangement of abbreviations. The corpus was created from the texts available on the Erasmus+ website using the in-built tools of the Sketch Engine interface and it includes a representative sample of 4 million words. The abbreviations were collected using the concordance queries which enable us to retrieve lemmas written with only capital letters (e.g. OLS for 'Online Linguistic Support') and subsequent manual filtering. The typology is based on previous works in this field (Fabijanić: 2015; Fabijanić: 2014a, 2014b; Malenica, Fabijanić: 2013; Fabijanić, Malenica: 2013), which provided a more consistent and more transparent approach to classification of abbreviations, based on different other works and approaches (Fandrych: 2008a; Fandrych: 2008b; Harley: 2006; López Rúa: 2006; Jackson, Ze Amvela: 2005; López Rúa: 2004; Plag: 2003; Plag: 2001; Stockwell, Minkova: 2001; Crystal: 1995; Algeo: 1991; Cannon: 1989). Abbreviations are classified according to two criteria: narrower and broader sense, and their differences in orthographic formation are described by the set of specific descriptors. The suggested description, classification and analysis were previously used in examining different terminologies and is used in this work to prove their applicability and sustainability.

Keywords: abbreviation, corpus, typology, EU, mobility, documents

\section{Introduction}

There has been a prominent increase in the production of abbreviations in the English language in the past several decades. This can be applied to both general language and the specialized jargons of various professions. In this paper, we

\footnotetext{
1 University of Zadar, Croatia, ivo.fabijanic@unizd.hr

2 University of Zadar, Croatia, fmalenica@unizd.hr
} 
provide a typological overview of abbreviations found in the corpus of Erasmus+ mobility documents, presented as an overview of classification and taxonomic arrangement of abbreviations. It was motivated by the exercise (learning/teaching material) preparation process for the Erasmus+ LanGuide project (KA2-HE-01/19) when the need arose to obtain a more profound understanding of formation patterns of abbreviations. The linguistic register of academic mobility abounds with abbreviations due to its bureaucratic nature, on the one hand, and the linguistic economy provided by the abbreviations, on the other hand. The corpus was created from the texts available on the Erasmus+ website (using the in-built tools of the Sketch Engine interface) and it includes a representative sample of 4 million words. The abbreviations were collected using the concordance queries which enable us to retrieve lemmas written with only capital letters (e.g. OLS for 'Online Linguistic Support') and subsequent manual filtering.

The typology of abbreviations is based on previous works in this field (Fabijanić 2014; Fabijanić 2015a and 2015b; Malenica and Fabijanić 2013; Fabijanić and Malenica 2013), which provided a more consistent and more transparent approach to the classification of abbreviations, based on different other works and approaches (Fandrych 2008a; Fandrych 2008b; Fandrych 2007; Gjuran-Coha and Bosnar-Valković 2008; Bieswanger 2007; Lehrer 2007; Harley 2006; López Rúa 2006; López Rúa 2004; Jackson and Ze Amvela 2005; Crystal 2004; Plag 2003; Stockwell and Minkova 2001; Crystal 1995; Algeo 1991; Cannon 1989). Abbreviations are classified according to two criteria: narrower and broader sense, and their differences in orthographic formation are described by the set of specific descriptors. The suggested description, classification and analysis were previously used in examining different terminologies and are used in this work to prove their applicability and sustainability. The corpus-based linguistic tools allow us to apply this descriptive apparatus on a representative sample of abbreviations, further annotate that sample and analyse its relevant word-formation features.

\section{Taxonomy of abbreviations}

López Rúa (2004 and 2006) divides the abbreviations into two groups of complex and simple shortenings, with the former divided into clippings, blends and initialisms, whereas the initialisms into alphabetisms and acronyms. Simple shortenings occur only in written form and encompass proper abbreviations. Almost all covered authors place blends and clippings into shortenings, with the exception of Jackson and Zé Amvela $(2005,88-89)$ who regard them separately from other abbreviations. 
López Rúa's approach is considered to be the most appropriate way of classifying abbreviations, especially the groups of acronyms and alphabetisms, for three reasons. Firstly, it is important for superordinate and subordinate terms to have different terminology. Secondly, the chosen terms should fit the properties of the named category; which in case of the term initialism denotes that words are created from initial letters of the constituent words and phrases, and the alphabetism denotes that the terms are pronounced letter-by-letter. Thirdly, despite their presence in written medium only, the group of proper abbreviations should not be ignored or confused with other types of abbreviations, as was the case not only with earlier dictionary practice, but also with some contemporary works as well.

According to López Rúa, an initialism is "(...) the result of selecting the initial letter, or occasionally the first two letters, of the orthographic words in a phrase and combining them to form a new sequence" (López Rúa 2006, 676). The two major ways for these words to be pronounced are as a word (prototypical acronyms) and as a series of letter names (prototypical alphabetisms) (López Rúa 2006,677 ). Examples of initialisms being pronounced in both ways can also be found, or even as a combination of the two, which are far less frequent ways of their pronunciation (Ibid.). Clipping is described as a "process by which a wordform of usually three or more syllables is shortened without a change in meaning or functions" (López Rúa 2006, 676). They are somewhat arbitrary regarding the part of the word that gets elided, and although they have informal connotations, there are examples of clippings replacing their source phrase (bus < omnibus). The morphological and phonetic properties of blends are a topic of numerous works (e.g. Gries 2004a and 2004b; Lehrer 2007; Crystal 2001; Fandrych 2007, 2008a and 2008b; López Rúa 2006 and 2007; Cannon 1989; Plag 2003). While there are numerous definitions of the term, López Rúa's definition was chosen for the purpose of this work, as her explanation is deemed sufficiently complex for this type of analysis. She states that that the blends are created by "(...) joining two or more word-forms through simple concatenation or overlap and then shortening at least one of them" $(2006,677)$.

This taxonomy is found to be the most appropriate one because it clearly distinguishes specific types of abbreviations, which means that it does not provide the same name for certain superordinate and/or subordinate terms, as it is an evident case in some authors. This in particular refers to initialisms, alphabetisms and acronyms, which are often used interchangeably or wrongly dubbed abbreviations or shortenings (e.g. in Plag 2003; Jackson and Zé Amvela 2005). Another argument for this usage of terms are the descriptive features of each abbreviation subcategory. The term initialism denotes an abbreviation created 
through usage of initial letters, applied to both alphabetisms and acronyms. The term alphabetism denotes an abbreviation pronounced as a series of letters of the alphabet, i.e. letter-by-letter, while the term acronym, coined in 1943, has been generally accepted to denote abbreviations pronounced as whole words.

\section{Methodology}

In order to analyse collect the data on use of abbreviations in the mobility-related documentation, a corpus of naturalistic data had to be collected for subsequent analysis. For this purpose, we chose the English version of the Erasmus+ website (https://ec.europa.eu/programmes/erasmus-plus/node_en). The corpus used in this paper was created via the Create corpus function in Sketch Engine. We used the in-built tool to retrieve the texts from the Erasmus+ website and the tool automatically compiled a corpus from it. The corpus based on the entire Erasmus+ website would be too large for our purposes (our estimate was 100-200 million words), which is why the corpus was built from a representative sample of roughly 4 million words. $^{3}$

To retrieve the abbreviations, we used the query in (1), which provides a list of all tokens written in uppercase letters. For practical purposes, we limited the query to 10 uppercase letters, because previous research (Fabijanić and Malenica 2013; Malenica and Fabijanić 2013) shows that these kinds of abbreviations are highly unlikely to occur. The CQL query in (1) also ignores the abbreviations written in lowercase letters (e.g. laser, sonar) or abbreviations in which only the first letter is capitalized (e.g. Nato). However, since these kinds of abbreviations are typically lexicalized abbreviations ${ }^{4}$ and generally not domain-specific, we believe that this minor concession would not present a significant obstacle for our research.

(1) [word="[[:upper:]]\{2,10\}"]

This query generated a list of 31,140 abbreviation tokens which were lemmatized and sorted by lemma frequency using Sketch Engine's in-built tools and downloaded in spreadsheet format for further annotation and filtering. The lemmatized and sorted list contained 286 abbreviations and was further filtered to remove the false positives. The false positives belonged to the following four groups:

\footnotetext{
3 The entire corpus contains 4,693,669 tokens (3,877,482 words).

4 The effects of lexicalization on use of upper- or lower-case letters in abbreviations is best visible in one of the most recent examples. When the term COVID-19 was first introduced in February 2020, it was spelled in all upper-case letters. As the term (and unfortunately the disease itself) became more widespread, alternative forms Covid-19 and simply covid became increasingly ubiquitous.
} 
a) words which were written in uppercase letters in the text for various reasons (emphasis, parts of headings, etc.), e.g. WHAT ELSE SHOULD YOU KNOW ABOUT THIS ACTION?;

b) abbreviations which are abbreviated only in written form but are pronounced as full words, e.g. $\mathrm{km}$ 'kilometre', EN 'English', PT 'Portugal, etc.

c) abbreviations which are not exclusive to the domain of academic mobility but other registers, e.g. ICT 'Information and Communication Technology', OJ 'Official Journal', or ACP 'African, Caribbean and Pacific';

d) abbreviations in other languages included in the Erasmus+ website, e.g. CIFE 'Centre International de Formation Européenne', SVE +Service Volontaire Européen', and SEPIE 'Servicio Español para la Internacionalización de la Educación'.

We additionally narrowed down our corpus to abbreviations whose normalized frequency was at least 1 per 1 million tokens. The final list analysed in this paper (in Appendix 1) included 122 abbreviations.

\section{Results and analysis}

In the first part of our analysis, we looked at the abbreviations by using the methodology presented in Fabijanić and Malenica (2013). Specifically, we looked at the creation of acronyms and alphabetisms in the broader and in the narrower sense. The narrower sense implies a symmetrical one-to-one relationship between the words in the source phrases and their initial graphemes which are used in the creation of a particular abbreviation. The broader sense entails the various deviations from the narrow pattern in terms of isomorphism between the abbreviation and its source phrase - omission of particular words of the source phrase, use of syllables in the creation of abbreviations, metathesis of graphemes, etc.

The analysis in this paper showed very little deviation from the prototypical pattern of creating abbreviations, i.e. creation of abbreviations in the narrower sense. Out of 122 abbreviations collected, 74 of them $(60.66 \%)$ belong to the abbreviations in the narrow sense (2a) and 48 (39.34\%) belong to the group of abbreviations in the broad sense. The majority of them $(\mathrm{N}=46)$ involve omission of particular words of the source phrase (2b), while only a small portion of them involve use of syllables in the creation of abbreviations (2c).

(2) a. ECAS

'European Commission Authentication System' ECEC

'Early Childhood Education and Care' 


$\begin{array}{ll}\begin{array}{l}\text { b. DCI } \\ \text { ICDE }\end{array} & \begin{array}{l}\text { 'Development and Cooperation Instrument' } \\ \text { 'International Council for Open and Distance Education' } \\ \text { c. ISCED }\end{array} \\ \text { EURES } & \text { 'International Standard Classification of Education' }\end{array}$

After the initial part of our analysis, we wanted to test two hypotheses which were formulated based on the data in Malenica and Fabijanić 2013; Fabijanić and Malenica 2013; and Malenica 2019. The first hypothesis we wanted to investigate was whether and to what extent the length of abbreviation affects its realization as acronym (pronounced as a single word) or as an alphabetism (pronounced letter-by-letter). Specifically, we hypothesized, following the data in Malenica and Fabijanić (2013), that the longer abbreviations are more likely to be realized as acronyms than as alphabetisms due to a linguistic economy principle (elaborated in Malenica 2019), with a cut-off point between the two types at around 3 or 4 graphemes.

In order to test this hypothesis, we collected the data on the length of abbreviations and their realization as an alphabetism or acronym. To verify that a particular abbreviation is pronounced as a whole word or in a letter-by-letter manner, we looked at the video and audio materials posted online by the relevant mobility institutions (e.g. a series of webinars organized by ENQA 'European Association for Quality Assurance in Higher Education'). Unfortunately, it was not possible to verify the pronunciation of all 122 abbreviations, but we believe the final total of 59 abbreviations analysed for this purpose is representative enough for our research.

In total, we found 19 acronyms with an average length of 4.58 graphemes $(S D=0.77, \min .=3, \max =6)$ and 40 alphabetisms with an average length of 3.1 graphemes $(S D=0.63, \min .=2, \max =5)$. These results are very much in line with the results in Malenica and Fabijanić (2013) in which a mean length of 4.49 graphemes was noted for acronyms and a mean length of 3.15 graphemes for alphabetisms. To test whether this difference in length between the two types of abbreviations is statistically significant, a binary logistic regression model was created with length of abbreviation as the predictor (independent) variable and the phonological realization (acronym or alphabetism) as a criterion (dependent) variable ${ }^{5}$, as seen in Figure 1 . The model proved to be significant $\left(X^{2}(57)=37.801\right.$, $\mathrm{p}<.001$ ) and a very good predictor of abbreviation type (McFadden $\mathrm{R}^{2}=0.510$ )

\footnotetext{
${ }^{5}$ The dataset meets the assumptions for using logistic regression listed in Clark-Carter (2018).
} 


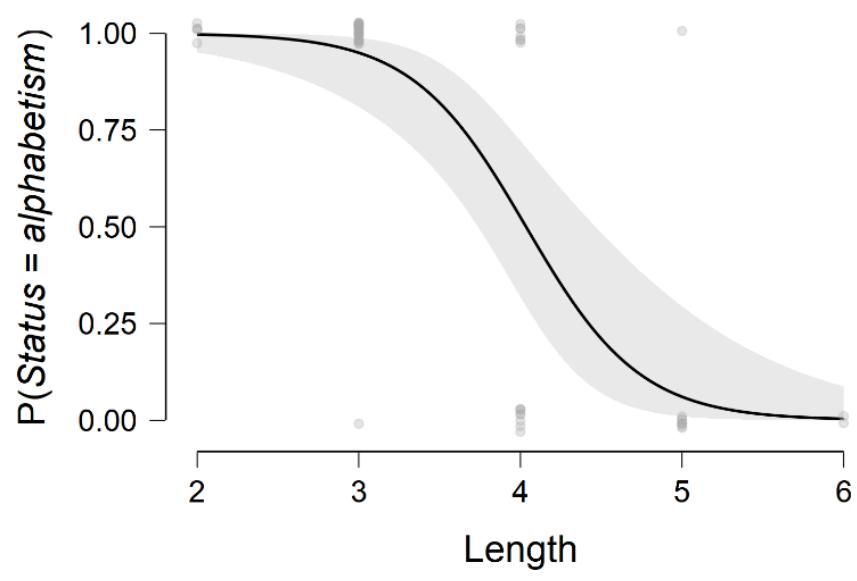

Figure 1. The binary logistic regression model with length of abbreviation as the predictor and type of abbreviation as the criterion variable

The second hypothesis we wanted to test was whether the omission of parts of the source phrases might be linked to the length of the abbreviation and their type. A chi-squared test showed a statistically significant correlation $\left(\chi^{2}{ }_{(1)}=18.344\right.$, $p<.001)$ between abbreviation type and use of ellipsis in their creation and the correlation was shown to be strong ( $\phi=0.567)$. In Table 1 , it is noticeable that acronyms are underrepresented in the Narrow Sense category, i.e. that a stronger preference for creation of abbreviations in the Broad Sense is more prominent among them and, conversely, that the alphabetisms are overrepresented in the Narrow Sense category. This confirms our hypothesis and shows that omission of particular elements of the source phrase (and other deviations from the general "one word one initial" principle) is more prominent with the formation of acronyms than with alphabetisms.

Table 1. Contingency table for abbreviation types and subtypes

\begin{tabular}{lcr} 
& Narrow & \multicolumn{1}{l}{ Broad } \\
\hline \hline Acronym & $3(10.42)$ & $15(7.58)$ \\
Alphabetism & $30(22.56)$ & $9(16.42)$
\end{tabular}

This result is further corroborated by the second binary logistic regression model created with the length of abbreviation as the predictor variable and the subtype of abbreviation (with or without ellipsis) as the dichotomous criterion variable. As can be seen in Figure 2, there is more variability within this model, meaning that the difference between the two groups is not as clear-cut as it was with the first model. 
However, this model also proved to be significant $\left(\chi^{2}(118)=31.583, p<.001\right.$, McFadden $\left.\mathrm{R}^{2}=0.198\right){ }^{6}$

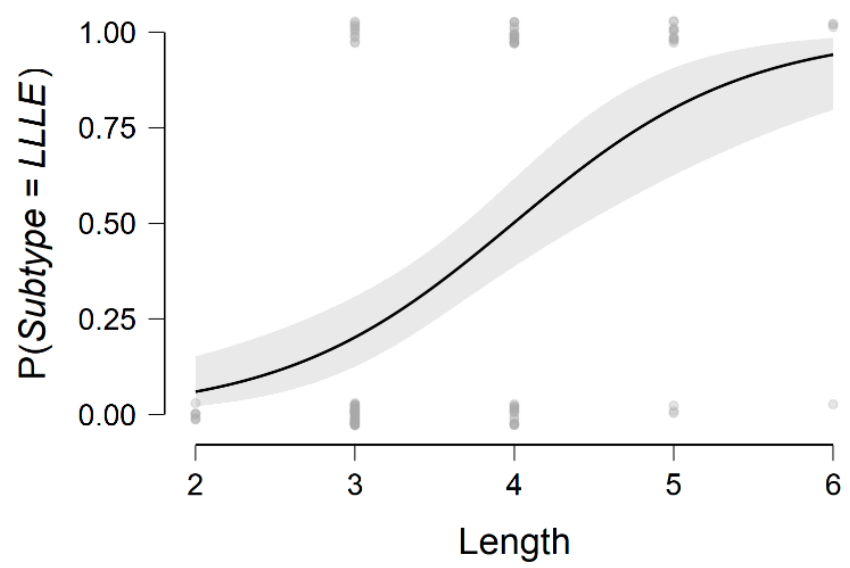

Figure 2. The binary logistic regression model with length of abbreviation as the predictor and subtype of abbreviation as the criterion variable

These results provide robust empirical confirmation that the formation of abbreviations is governed by the principles of linguistic economy. Specifically, the data indicate that when a potential new abbreviation is created, a form with over three initials (i.e. three syllables when pronounced word-by-word) is deemed uneconomical, which is why the whole-word pronunciation is more likely to be used (Figure 1.). The data collected in this paper are consistent with the data collected in previous research (cf. Malenica and Fabijanić 2013; Malenica 2019), but the reason why the cut-off point between the "more" and "less" economical forms is 3 syllables is not clear at this stage. A higher proportion of omitted source phrase elements among acronyms (Table 1) and among longer abbreviations (Figure 2) indicates that this strategy is often followed by certain trade-offs in terms of deviations from the "prototypical" mode of creation of abbreviations. Other factors like the ease of pronunciation most probably play a role in this (cf. Malenica 2019), but these matters are beyond the scope of this paper.

\footnotetext{
${ }^{6}$ For the sake of maintaining the binary logistic regression model, the two abbreviations which do not involve omission of source phrase words but are classified as abbreviations in the broader sense are not included in the model.
} 


\section{Conclusion}

Our aim in this paper was to provide a brief overview of the typological system used for classifying abbreviations from the field of mobility and education and analyse several factors which influence their formation. We used the corpus-based methods to collect a representative sample of abbreviations from this domain and have demonstrated the applicability of the typological system presented in earlier works (Fabijanić 2014; Fabijanić 2015a, 2015b; Malenica and Fabijanić 2013; Fabijanić and Malenica 2013) on a new corpus of abbreviations. The analysis of the corpus of about 4 million tokens revealed that the majority (60.66\%) of abbreviations in the corpus are created by adhering to the "narrow" sense template, meaning every word of the source phrase is represented by a single grapheme of the abbreviation. However, a relatively large portion of the sample $(39.34 \%)$ deviates from this prototype, and these deviations include omission of particular words from the source phrase or use of syllables (see section 4.). Further analysis showed that the formation of acronyms is strongly correlated with longer source phrases and, consequently, longer abbreviations (over 3 syllables), while the formation of alphabetisms is more commonly associated with shorter abbreviations (3 syllables or less). Acronyms were also shown to be more strongly associated with the omission of source phrase elements and other types of deviations from the prototypical template for forming abbreviations. We believe the data presented in this paper provide strong evidence in favour of the proposed classificatory system and further demonstrate the importance of the linguistic economy principle for the formation of abbreviations. We believe further research in the field would lead to more complex models of formation which would include factors such as ease of pronunciation or practicality of abbreviation and we hope our work will stimulate research heading in this direction.

\section{Acknowledgement}

This research was supported by the Erasmus+ grant number 19-203-060377 - KA2$\mathrm{HE}-01 / 19$.

\section{References}

Algeo, John (ed.). 1991. Fifty Years Among the New Words. Cambridge: Cambridge University Press. 
Bieswanger, Markus. 2007. "2 abbrevi8 or not 2 abbrevi8: A contrastive analysis of different space and time-saving strategies in English and German text messages." Texas Linguistic Forum, Vol. 50. Retrieved from: http://salsa.ling.utexas.edu/proceedings/2006/Bieswanger.pdf

Cannon, Garland. 1989. "Abbreviations and Acronyms in English Word-Formation." American Speech 64(2), 99-127.

Clark-Carter, David. 2019. Quantitative psychological research: The complete student's companion (4th Edition). Abingdon: Routledge.

Crystal, David. 1995. The Cambridge Encyclopaedia of the English Language. Cambridge: Cambridge University Press.

Crystal, David. 2004. A Glossary of Netspeak and Textspeak. Edinburgh: Edinburgh University Press.

Fabijanić, Ivo. 2014. "Dictionary of Abbreviations in Linguistics: Towards Defining Cognitive Aspects as Structural Elements of the Entry." In Proceedings of the XVI EURALEX International Congress: The User in Focus, ed. by Andrea Abel, Chiara Vettori, Natascia Ralli, 145-168. Institute for Specialised Communication and Multilingualism: Bolzano.

Fabijanić, Ivo. 2015a. "A Dictionary of Abbreviations in Linguistics: Towards a Bilingual, Specialized, Single-field, Explanatory Dictionary." In Planning Nonexistent Dictionaries, ed. by João Paulo Silvestre and Alina Villalva, 113-133. Lisabon, Centro de Linguística da Universidade de Lisboa - Universidade de Aveiro.

Fabijanić, Ivo. 2015b. "Dictionary of Abbreviations in Linguistics: Towards Defining Functional Aspects as Structural Elements of the Entry". In The Practice of Foreign Language Teaching: Theories and Applications, ed. by Azamat Akbarov, 278-288. Newcastle upon Tyne: Cambridge Scholars Publishing.

Fabijanić, Ivo and Frane Malenica. 2013. "Abbreviations in English medical terminology and their adaptation to Croatian." JAHR - Godišnjak Katedre za društvene i humanističke znanosti u medicini Sveučilišta u Rijeci 4 (7), 71-105.

Fandrych, Ingrid. 2008a. "Pagad, Chillax and Jozi: A Multi-Level Approach to Acronyms, Blends, and Clippings." Nawa Journal of Language and Communication 2 (2), 71-88.

Fandrych, Ingrid. 2008b. "Submorphemic elements in the formation of acronyms, blends and clippings." Lexis 2: "Lexical Submophemics / La submorphémique lexicale" 105-123.

Fandrych, Ingrid. 2007. "Electronic Communication and Technical Terminology: A Rapproachment?" Nawa Journal of Language and Communication 1 (1), 147-158.

Gjuran-Coha, Anamarija and Brigita Bosnar-Valković. 2008. "Uporaba kratica u jeziku medicinske struke." Filologija 50, 1-12. 
Harley, Heidi. 2006. English Words: A Linguistic Introduction. Oxford: Blackwell Publishing.

Jackson, Howard and Etienne Zé Amvela. 2005. Words, Meaning and Vocabulary. London: Continuum.

Lehrer, Adrienne. 2007. "Blendalicious." In Lexical Creativity. Text and Context, ed. by Judith Munat, 115-133. Amsterdam: John Benjamins.

López Rúa, Paula. 2004. "Acronyms \& Co.: A typology of typologies". Estudios Ingleses de la Universidad Complutense 12, 109-129.

López Rúa, Paula. 2006. "Non-Morphological Word Formation." Encyclopedia of Language and Linguistics (2nd Edition) 2, 675-678. Oxford: Elsevier.

Malenica, Frane and Ivo Fabijanić. 2013. "Abbreviations in English Military Terminology." Brno Studies in English 39(1), 59-87.

Malenica, Frane. 2019. "From Writing to Speech - an Optimality Theoretic Approach to Formation of Acronyms in the English Language". In Migrations: Literary and Linguistic Aspects, ed. by Ivo Fabijanić, Lidija Štrmelj, Vesna Ukić Košta, and Monika Bregović, 369-394. Berlin: Peter Lang GmbH.

Plag, Ingo. 2003. Word-formation in English. Cambridge: Cambridge University Press.

Stockwell, Robert and Donka Minkova. 2001. English Words: History and Structure. Cambridge: Cambridge University Press. 


\section{APPENDIX}

Appendix 1. Corpus of abbreviations

\begin{tabular}{|c|c|c|c|}
\hline $\begin{array}{l}\text { Abbre- } \\
\text { viation }\end{array}$ & Full phrase & $\begin{array}{c}\text { Normalized } \\
\text { freq. }\end{array}$ & $\begin{array}{l}\text { Sub } \\
\text { type }\end{array}$ \\
\hline $\mathrm{AE}$ & Adult Education & 24.71 & LLL \\
\hline AIPY & Agency for International Programs for Youth & 5.33 & LLLE \\
\hline APV & Advance Planning Visit & 45.17 & LLL \\
\hline AWP & Annual Work Programme & 2.13 & LLL \\
\hline CAWI & Computer Assisted Web Interview & 6.82 & LLL \\
\hline CBHE & Capacity Building in Higher Education & 6.18 & LLLE \\
\hline CEEPUS & Central European Exchange Programme for University Studies & 3.83 & LLLE \\
\hline CEERES & Central and East European, Russian and Eurasian Studies & 1.7 & LLLE \\
\hline CLIL & Content and Language Integrated Learning & 6.6 & LLLE \\
\hline CRELL & Centre for Research on Lifelong Learning & 8.52 & LLLE \\
\hline CSS & Central Support Service & 15.98 & LLL \\
\hline CVET & continuing vocational education and training & 3.41 & LLLE \\
\hline $\mathrm{DCl}$ & Development and Cooperation Instrument & 60.72 & LLLE \\
\hline DEOR & Dissemination and exploitation of results & 4.05 & LLLE \\
\hline DG & Directorate General & 88.63 & LLL \\
\hline DGT & Directorate-General for Translation & 9.59 & LLLE \\
\hline EAC & Education and Culture & 176.83 & LLLE \\
\hline EACEA & Education, Audiovisual and Culture Executive Agency & 265.68 & LLLE \\
\hline EASQ & European Area of Skills and Qualifications & 2.34 & LLLE \\
\hline EC & European Commission & 113.98 & LLL \\
\hline ECAS & European Commission Authentication System & 12.57 & LLL \\
\hline ECEC & Early Childhood Education and Care & 21.94 & LLL \\
\hline ECHE & Erasmus Charter for Higher Education & 227.33 & LLL \\
\hline ECTS & European Credit Transfer and Accumulation System & 103.97 & LLLE \\
\hline ECVET & European Credit System for Vocational Education and Training & 152.33 & LLLE \\
\hline EDF & European Development Fund & 67.54 & LLL \\
\hline EENEE & European Expert Network on Economics of Education & 13.85 & LLLE \\
\hline EHEA & European Higher Education Area & 32.17 & LLL \\
\hline EIF & European Investment Fund & 17.47 & LLL \\
\hline
\end{tabular}




\begin{tabular}{|c|c|c|c|}
\hline $\begin{array}{l}\text { Abbre- } \\
\text { viation }\end{array}$ & Full phrase & $\begin{array}{c}\text { Normalized } \\
\text { freq. }\end{array}$ & $\begin{array}{l}\text { Sub } \\
\text { type }\end{array}$ \\
\hline EIPA & European Institute of Public Administration & 4.26 & LLLE \\
\hline ELGPN & European Lifelong Guidance Policy Network & 1.28 & LLL \\
\hline EMJMD & Erasmus Mundus Joint Master Degree & 455.29 & LLL \\
\hline EMMC & Erasmus Mundus Master Courses & 7.03 & LLL \\
\hline EMT & European Master's in Translation & 10.01 & LLLE \\
\hline EMT & Executive Agency online Mobility Tool & & LLLE \\
\hline ENI & European Neighbourhood Instrument & 53.26 & LLL \\
\hline ENIC & European Network of Information Centers & 1.92 & LLLE \\
\hline ENP & European Neighbourhood Policy & 17.9 & LLL \\
\hline ENQA & $\begin{array}{l}\text { European Association for Quality Assurance in Higher } \\
\text { Education }\end{array}$ & 3.62 & LLLE \\
\hline EPALE & Electronic Platform for Adult Learning in Europe & 81.39 & LLLE \\
\hline EPRP & Erasmus+ Project Results Platform & 1.07 & LLL \\
\hline EQAR & European Quality Assurance Register & 6.6 & LLL \\
\hline EQAVET & $\begin{array}{l}\text { European Quality Assurance in Vocational Education and } \\
\text { Training }\end{array}$ & 73.93 & LLLE \\
\hline EQF & European Qualifications Framework & 119.52 & LLL \\
\hline ESAA & Erasmus+ Student and Alumni Association & 1.07 & LLLE \\
\hline ESF & European Social Fund & 8.74 & LLL \\
\hline ESL & early school leaving & 5.54 & LLL \\
\hline ESN & Erasmus Student Network & 2.56 & LLL \\
\hline ETER & European Tertiary Education Register & 2.13 & LLL \\
\hline ETF & European Training Foundation & 2.13 & LLL \\
\hline EU & European Union & 2341.24 & LLL \\
\hline EUI & European University Institute & 4.05 & LLL \\
\hline EUNIC & EU National Institutes for Culture & 2.13 & LLLE \\
\hline EUPA & European Union Programmes Agency & 6.82 & LLL \\
\hline EURES & European Employment Services & 4.9 & SLLL \\
\hline EVS & European Voluntary Service & 234.78 & LLL \\
\hline EWS & Early Warning System & 3.2 & LLL \\
\hline EYCA & European Youth Card Association & 1.7 & LLL \\
\hline
\end{tabular}




\begin{tabular}{|c|c|c|c|}
\hline $\begin{array}{l}\text { Abbre- } \\
\text { viation }\end{array}$ & Full phrase & $\begin{array}{c}\text { Normalized } \\
\text { freq. }\end{array}$ & $\begin{array}{l}\text { Sub } \\
\text { type }\end{array}$ \\
\hline EYP & European Youth Portal & 2.13 & LLL \\
\hline FPA & Framework Partnership Agreement & 9.37 & LLL \\
\hline FWC & Framework Contracts & 1.07 & LLL \\
\hline GTSET & Grimsby Town Sports and Education Trust & 1.7 & LLLE \\
\hline HEA & Higher Education Authority & 4.47 & LLL \\
\hline HEI & Higher education institution & 337.48 & LLL \\
\hline HRD & Human Resources Development & 1.7 & LLL \\
\hline HRDA & Human Resource Development Authority & 1.7 & LLL \\
\hline $\mathrm{IAB}$ & Independent Audit Body & 1.28 & LLL \\
\hline ICCS & International Civic and Citizenship Education Study & 1.28 & LLLE \\
\hline ICDE & International Council for Open and Distance Education & 7.46 & LLLE \\
\hline ICILS & International Computer and Information Literacy Study & 4.69 & LLLE \\
\hline ICM & International Credit Mobility & 15.98 & LLL \\
\hline IEG & International Erasmus Games & 1.28 & LLL \\
\hline IMIM & International Master in Innovative Medicine & 1.28 & LLLE \\
\hline IPA & Instrument for Pre-accession Assistance & 32.38 & LLLE \\
\hline IPR & Intellectual Property Rights & 7.03 & LLL \\
\hline IPTS & Institute for Prospective Technological Studies & 4.05 & LLLE \\
\hline IRO & International Relations Office & 1.92 & LLL \\
\hline ISCED & International Standard Classification of Education & 10.23 & SLLL \\
\hline ISP & Intensive Study Programme & 20.24 & LLL \\
\hline IVET & Initial Vocational Education and Training & 4.47 & LLLE \\
\hline $\mathrm{JM}$ & Jean Monnet & 11.08 & LLL \\
\hline JMD & Joint Master Degree & 3.41 & LLL \\
\hline JRC & Joint Research Centre & 17.68 & LLL \\
\hline LDV & Leonardo da Vinci & 1.92 & LLL \\
\hline LLL & Lifelong Learning & 10.01 & LLL \\
\hline LLP & Lifelong Learning Programme & 171.29 & LLL \\
\hline MCAST & Malta College of Arts, Science and Technology & 1.07 & LLLE \\
\hline MFF & Multi-annual Financial Framework & 4.47 & LLLE \\
\hline MOEC & Ministry of Education and Culture & 1.28 & LLL \\
\hline
\end{tabular}




\begin{tabular}{|c|c|c|c|}
\hline $\begin{array}{l}\text { Abbre- } \\
\text { viation }\end{array}$ & Full phrase & $\begin{array}{c}\text { Normalized } \\
\text { freq. }\end{array}$ & $\begin{array}{l}\text { Sub } \\
\text { type }\end{array}$ \\
\hline NARIC & National Academic Recognition Information Centre & 41.12 & LLL \\
\hline NCP & National Coordination Points & 2.77 & LLL \\
\hline NEET & Not in Employment, Education or Training & 5.11 & LLLE \\
\hline NEO & National Erasmus+ Office & 9.37 & LLL \\
\hline NESET & $\begin{array}{l}\text { Network of Experts working on the Social dimension of } \\
\text { Education and Training }\end{array}$ & 12.36 & LLLE \\
\hline NQF & National Qualifications Framework & 4.26 & LLL \\
\hline NSLE & National School for Leadership in Education & 1.28 & LLLE \\
\hline NSS & National Support Services & 1.28 & LLL \\
\hline OECD & Organisation for Economic Co-operation and Development & 69.88 & LLLE \\
\hline OER & Open Educational Resources & 101.84 & LLL \\
\hline OHER & On-line Higher Education Report & 1.7 & LLL \\
\hline OID & Organisation ID & 1.7 & LLL \\
\hline OLS & Online Linguistic Support & 59.65 & LLL \\
\hline OMC & Open Method of Coordination & 10.65 & LLLE \\
\hline OP & Operational Programme & 3.83 & LLL \\
\hline PBL & Problem Based Learning & 1.92 & LLL \\
\hline PIAAC & $\begin{array}{l}\text { Programme for the International Assessment of Adult } \\
\text { Competencies }\end{array}$ & 18.54 & LLLE \\
\hline PID & Project Implementation Directorate & 1.28 & LLL \\
\hline PLL & Programme for Lifelong Learning & 3.83 & LLLE \\
\hline PLM & People in the labour market & 3.2 & LLLE \\
\hline PSA & Partner Support Agencies & 19.17 & LLL \\
\hline RTT & Researchers, teachers and trainers & 6.82 & LLLE \\
\hline SALTO & Support, Advanced Learning and Training Opportunities & 61.15 & LLLE \\
\hline $\mathrm{SCHE}$ & Short Cycles Higher Education & 6.82 & LLL \\
\hline SEG & School Education Gateway & 7.46 & LLL \\
\hline SGIB & Standing Group on Indicators and Benchmarks & 1.28 & LLLE \\
\hline SOPHRD & $\begin{array}{l}\text { Sectoral Operational Programme Human Resource } \\
\text { Development }\end{array}$ & 1.07 & LLL \\
\hline SSA & Sector Skills Alliances & 2.13 & LLL \\
\hline TC & Technical Committee & 1.28 & LLL \\
\hline
\end{tabular}




\begin{tabular}{|l|l|r|r|}
\hline $\begin{array}{l}\text { Abbre- } \\
\text { viation }\end{array}$ & \multicolumn{1}{|c|}{ Full phrase } & $\begin{array}{c}\text { Normalized } \\
\text { freq. }\end{array}$ & $\begin{array}{c}\text { Sub } \\
\text { type }\end{array}$ \\
\hline TCA & Transnational Cooperation Activities & 25.99 & LLL \\
\hline URF & Unique Registration Facility & 9.16 & LLL \\
\hline WBAA & Western Balkans Alumni Association & 2.34 & LLL \\
\hline WPI & Work Programme Index & 208.15 & LLL \\
\hline
\end{tabular}

LLL = 1 source word represented by 1 grapheme in abbreviation letter;

LLLE = 1 source word represented by 1 grapheme in abbreviation letter with omission of words in the source phrase;

LLLE $=1$ source word represented by 1 grapheme in abbreviation letter with use of syllables in the abbreviation. 